\title{
Redução de Desvios de Previsão Climática usando Filtragem Adaptativa no Domínio da Frequência
}

\author{
Leandro Ferreira Gentile Pinto, Michel Pompeu Tcheou, Lisandro Lovisolo, Sin Chan Chou.
}

\begin{abstract}
Resumo-Este trabalho aborda o uso de filtros adaptativos no domínio da frequência, via algoritmo RLS (Recursive Least Squares) e DCT-2D (Two-dimensional Discrete Cosine Transform), com o objetivo de reduzir desvios de previsão climática produzidos por um modelo regional climático. As previsões do modelo regional (modelo Eta) são comparadas com os dados observados (reanálises do NCEP). As variáveis climáticas consideradas são as componentes zonal e meridional do vento, altura geopotencial e umidade específica, produzidas pelo modelo sazonal Eta, na resolução espacial de $40 \mathrm{~km}$. Os resultados indicam que a aplicação proposta é capaz de reduzir métricas de desvio, como o erro quadrático médio e o erro máximo, contribuindo com melhores previsões climáticas.
\end{abstract}

Palavras-Chave-Filtragem adaptativa, algoritmo RLS, DCT2D, previsão climática.

Abstract-This work aims at using adaptive filters in the frequency domain, through the RLS (Recursive Least Squares) algorithm and DCT-2D (Two-dimensional Discrete Cosine Transform), with the objective of reducing deviations of climatic forecasts produced by a regional climate model. The regional model predictions (Eta model) are compared with the observed data (NCEP reanalysis). The climatic variables considered are the zonal and meridional components of the wind, geopotential height and specific humidity, produced by the Eta seasonal model, in the spatial resolution of $\mathbf{4 0} \mathrm{km}$. The results indicate that the proposed application is capable to reduce deviation metrics, such as the mean square error and the maximum error, contributing to better climate predictions.

Keywords-Adaptive filtering, RLS algorithm, DCT-2D, climatic forecast.

\section{INTRODUÇÃo}

Apesar do crescente uso da previsão climática numérica, erros de previsão estão presentes. Neste trabalho consideramos o problema da correção de desvios de previsão numérica utilizando filtros adaptativos. Mais especificamente, as previsões fornecidas pelo modelo atmosférico regional Eta [1] (adotado pelo CPTEC/INPE - Centro de Previsão de Tempo e Estudos Climáticos/Instituto Nacional de Pesquisas Espaciais) são comparadas com dados de reanálise climática. Essa comparação permite avaliar a qualidade da previsão. $\mathrm{O}$ processo de ajuste de previsões usando filtros adaptativos é aplicado no domínio da frequência, diferentemente de [2].

Os modelos de previsão numérica decompõem a atmosfera em células (volumes) e modelando numericamente as interações físicas entre as células, avaliam a evolução do

Leandro Ferreira Gentile Pinto, Michel Pompeu Tcheou, Lisandro Lovisolo, Programa de Pós-Graduação em Engenharia Eletrônica, Universidade do Estado do Rio de Janeiro, Rio de Janeiro-RJ, Brasil, e Sin Chan Chou, Instituto Nacional de Pesquisas Espaciais, Cachoeira Paulista-SP, Brasil E-mails: leandrogentile.lg@gmail.com,mtcheou@uerj.br, lisandro@uerj.br, chou.sinchan@cptec.inpe.br estado da atmosfera utilizando métodos numéricos. A previsão climática fornece as variáveis prognósticas: componentes zonal e meridional do vento, temperatura do ar, umidade e temperatura do solo, água líquida ou gelo das nuvens, umidade específica, pressão à superfície e energia cinética turbulenta [3]. Para produzí-las, além do modelo numérico, são necessárias condições de contorno e iniciais [4]. O modelo de previsão climática Eta [1] utiliza como condição inicial os dados do modelo global GFS (Global Forecast System) [5], fornecidos pelo NCEP (National Centers for Environmental Prediction dos EUA) e como condições de contorno, os dados fornecidos pelo modelo global de circulação geral atmosférico (MCGA) do CPTEC [4]. Neste trabalho, consideramos uma resolução espacial de $40 \mathrm{~km}$.

O NCEP fornece um conjunto de dados que representam com maior acurácia as condições climáticas observadas; tal conjunto de dados é chamado de reanálise NCEP/CFSR (Climate Forecast System Reanalysis) [6]. A reanálise NCEP é um conjunto de dados meteorológicos, organizados numa grade de cobertura global representando a evolução do estado atmosférico da Terra.

Dados atmosféricos oriundos de modelos numéricos são por si volumétricos; temos a resolução sobre a superfície terrestre e em função da altura de forma a fornecer células atmosféricas em função de latitude, longitude e altitude para as quais obtêmse as variáveis prognósticas. Além disso, essas variáveis são fornecidas para um intervalo de tempo, em geral fixo de algumas horas. Temos assim dados volumétricos discretos no tempo. Por outro lado, o valor de uma variável atmosférica em uma dada altitude numa determinada região da superfície terrestre pode ser representada como uma imagem digital, para cada pixel dessa imagem há uma coordenada geográfica (em função da latitude e longitude) associada.

Assim, empregando os dados do NCEP como referência, podemos avaliar o erro da previsão climática fornecida pelo Eta. Consequentemente, neste trabalho, empregamos filtros adaptativos com a finalidade de reduzir erros de previsão numérica climática. Os filtros são aplicados no domínio da frequência através da DCT (Discrete Cosine Transform). Entende-se que a correção das componentes espectrais, em vez dos valores espaciais (pixeis), permitiria corrigir os modos implícitos ao modelo matemático empregado para a previsão.

A Figura 2(a) apresenta a região na qual é aplicada a proposta, que engloba a América do Sul, partes da América Central e África. Os vértices da Figura 2(a) representam a região onde são inseridas as condições de contorno. As coordenadas dos vértices são $115^{\circ} \mathrm{W}$ e $30^{\circ} \mathrm{N}, 115^{\circ} \mathrm{W}$ e $58^{\circ} \mathrm{S}$, $6^{\circ} \mathrm{W}$ e $30^{\circ} \mathrm{N}$ e $6^{\circ} \mathrm{W}$ e $58^{\circ} \mathrm{S}$. 


\section{Metodologia Proposta}

A Figura 1 ilustra a metodologia empregada nos anos de treinamento e ajuste, para o processamento de uma variável prognóstica, enfatizando o uso da filtragem adaptativa no domínio da frequência. Os dados do NCEP e do Eta correspondem a séries temporais para um ponto (coordenada geográfica), para diversas variáveis prognósticas - a Figura 1 apresenta o processamento para uma variável prognóstica. A interpolação bidimensional redimensiona os dados do Eta para a resolução do NCEP. A filtragem adaptativa requer treinamento. Os filtros alteram as componentes espectrais da previsão, por isso são aplicados após a DCT bidimensional (DCT-2D) e seu arranjo através de um varredura zig-zag e o consequente agrupamento em regiões frequenciais. Os filtros RLS (Recursive Least Squares) [7], [8] são então projetados para atuar em subconjuntos de componentes espectrais. Os filtros FIR (Finite Impulse Response) [9] projetados adaptativamente são usados para ajustar a previsão obtida com o Eta no domínio da DCT-2D. A varredura inversa zig-zag reordena os coeficientes e finalmente aplica-se a Transformada Inversa Discreta do Cosseno Bidimensional (IDCT2D, Two-dimensional Inverse Discrete Cosine Transform) para a obtenção da variável prognóstica Eta ajustada. Consideramos o ajuste de previsões climáticas entre os anos 2008 e 2010. Filtros são projetados/treinados para corrigir a previsão do ano XX usando os dados correspondentes ao ano (XX-1). Por exemplo, se queremos ajustar o ano de 2009, usamos o ano de 2008 para gerar o conjunto de treinamento.

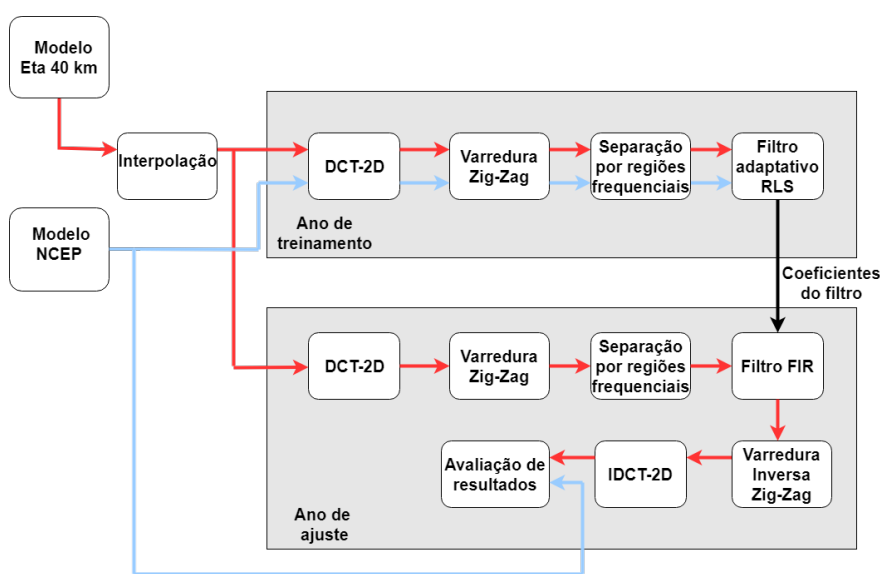

Fig. 1. Esquema da utilização de filtros adaptativos no domínio da frequência.

\section{A. Interpolação Bidimensional}

A diferença entre as grades (amostragem espacial em termos de latitude e longitude) do modelo Eta e das reanálises do NCEP requerem o ajuste dos dois modelos para uma grade comum. A uma determinada altitude (ou nível de pressão atmosférica) os dados do modelo Eta possuem $220 \times 275$ elementos e os do NCEP $177 \times 219$ elementos na região da Figura 2(a). Isto é, o modelo Eta utiliza uma resolução de $0,4^{\circ}$, enquanto o NCEP é disponibilizado com resolução de $0,5^{\circ}$. Assim, aplica-se uma interpolação bidimensional para redimensionar os dados do Eta de forma que em cada nível de pressão haja, $177 \times 219=38.763$ elementos.
A interpolação bidimensional implementada emprega Splines - funções formadas por polinômios de ordem menores ou iguais a $m$, definidos para cada intervalo entre os pontos de interpolação de modo que em cada ponto de interpolação o spline é contínuo [10]. No caso, emprega-se uma Spline cúbica, assim, o valor interpolado em um ponto utiliza uma interpolação cúbica em cada dimensão.

\section{B. Análise Espectral Bidimensional}

Uma imagem, um conjunto ou um bloco de dimensão $M \times N$ contêm dados de amplitude indexados espacialmente. A Transformada Discreta do Cosseno Bidimensional DCT-2D (Discrete Cosine Transform) desse conjunto permite converter os dados de amplitude espacial em componentes representando frequências espaciais. A DCT-2D de uma matriz $\mathbf{U}$ de tamanho $M \times N$ resulta em uma matriz $\mathbf{G}$, cujos elementos são definidos por

$g_{p, q}=c_{p} c_{q} \sum_{m=0}^{M-1} \sum_{n=0}^{N-1} u_{m, n} \cos \left[\frac{\pi p}{M}\left(m+\frac{1}{2}\right)\right] \cos \left[\frac{\pi q}{N}\left(n+\frac{1}{2}\right)\right]$,

com $0 \leq p \leq M-1$ e $0 \leq q \leq N-1$, e onde $u_{m, n}$ representa o elemento na $m$-ésima linha e $n$-ésima coluna da matriz $\mathbf{U}$ e $g_{p, q}$ o valor na $p$-ésima linha e $q$-ésima coluna da matriz $\mathbf{G}$. Os valores de $p$ e $q$ determinam a frequência dos elementos de G, os argumentos dos cossenos na equação (1). As constantes de normalização $c_{p}$ e $c_{q}$ dependem das quantidades de linhas $M$ e colunas $N$ de $\mathbf{U}$, respectivamente [11].

A DCT-2D tem sido empregada na codificação de imagens, pois, em geral, permite concentrar grande fração da energia de imagens naturais em $p$ e $q$ pequenos, em frequências espaciais baixas, da mesma forma também ocorre para os dados climáticos. A Figura 2 apresenta a DCT-2D 2(b) de uma variável prognóstica do modelo Eta num nível de pressão 2(a).

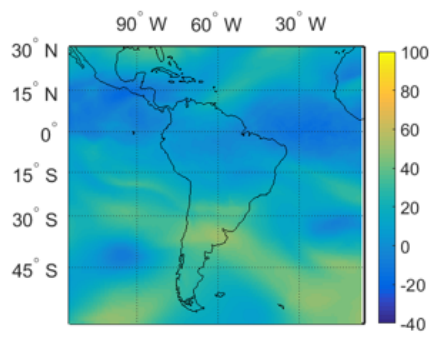

(a)

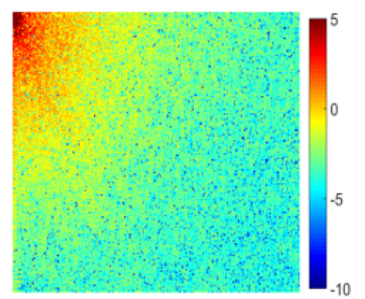

(b)
Fig. 2. Exemplo de variável prognóstica (componente zonal do vento em $\mathrm{m} / \mathrm{s}$ ) gerada pelo Eta para um determinado nível de pressão (altura atmosférica) em (a) e (b) é a sua DCT-2D, com frequências espaciais (coeficientes) em ciclos $/ \mathrm{km}$

\section{Varredura Zig-Zag}

A varredura zig-zag ordena crescentemente os coeficientes da DCT-2D em função da frequência correspondente aos elementos $\left(\omega=\left(p^{2}+q^{2}\right)^{1 / 2}\right)$. Em geral, isso corresponde a organizar os coeficientes em ordem decrescente de energia. Como resultado deste procedimento obtém-se um vetor no qual podemos dividir os coeficientes em regiões frequenciais, agrupando os coeficientes da DCT-2D por faixas de baixa, 610 
média e alta frequência que são usados para a filtragem adaptativa. A varredura zig-zag é aplicada em todas as matrizes de coeficientes da DCT-2D, gerando vetores de 38.763 elementos.

\section{Agrupamento dos Dados no Domínio Espectral}

Os elementos da DCT-2D são agrupados conforme ilustrado na Figura 3. A região 1 contém 3.600 coeficientes, a região 2 tem 16.000 coeficientes e a região 4 é composta por 19.163 coeficientes. É importante ressaltar que o projeto de filtros adaptativos é realizado na região 1. Nas regiões 2 e 3 , os valores dos coeficientes são muito próximos de zero, tornando-se irrelevante a aplicação da filtragem adaptativa nestas regiões, o que colabora para economia de bits e maior velocidade computacional.

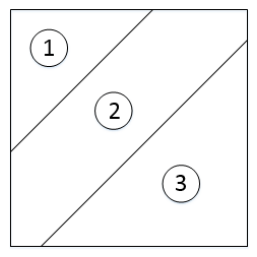

Fig. 3. Coeficientes da DCT-2D separados por regiões frequenciais.

\section{E. Projeto de Filtros para o Ajuste das Componentes Espec- trais}

Este trabalho emprega filtros adaptativos de forma a melhorar a previsão sazonal do Eta em comparação com às reanálises do NCEP. A filtragem adaptativa é aplicada no processamento de sinais pois proporciona flexibilidade e desempenho relevantes em diversas tarefas [7], [8]. A Figura 4 ilustra um sistema adaptativo; $d(n)$ é o sinal de referência, $\mathbf{x}(n)=[x(n) \quad x(n-1)$ $\left.\begin{array}{lll}x(n-2) & \ldots & x(n-N)\end{array}\right]^{T}$ é o vetor do sinal de entrada, w(n) é o vetor de coeficientes do filtro adaptativo e $N$ é a ordem do filtro adaptativo. A saída do filtro adaptativo é definida por

$$
y(n)=\sum_{i=0}^{N} w_{i}(n) x(n-i)=\mathbf{w}^{T}(n) \mathbf{x}(n),
$$

onde $\mathbf{w}(n)=\left[\begin{array}{llll}w_{0}(n) & w_{1}(n) & \ldots & w_{N}(n)\end{array}\right]^{T}$. O símbolo $T$ indica a transposição da matriz. A saída do filtro adaptativo deve seguir o sinal de referência $d(n)$ e podemos definir o erro $e(n)=d(n)-y(n) . \mathrm{O}$ algoritmo adaptativo é responsável pelo ajuste dos coeficientes do filtro, visando a redução do erro quadrático.

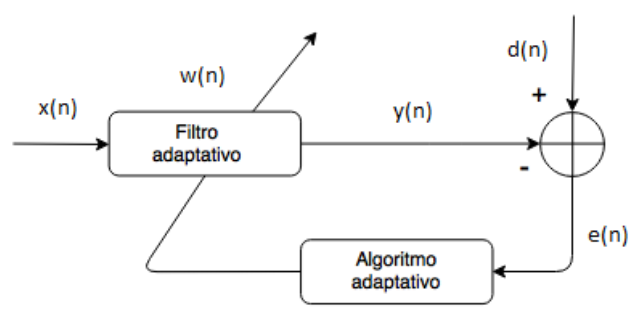

Fig. 4. Sistema adaptativo.
1) Algoritmo RLS (Recursive Least Squares): Para atualização dos coeficientes do filtro, isto é, o projeto de filtros para redução do erro de previsão climática, empregamos o algoritmo RLS pois apresenta bom desempenho para sistemas sujeitos a rápidas variações na amplitude do sinal [7]. Os coeficientes do filtro w são atualizados a cada iteração $n$ (nova entrada de dados) usando [7]

$$
\begin{aligned}
\mathbf{w}(n) & =\mathbf{R}_{D}^{-1}(n) \mathbf{p}_{D}(n) \\
\mathbf{R}_{D}(n) & =\sum_{i=0}^{n} \lambda^{(n-i)} \mathbf{x}(i) \mathbf{x}^{T}(i) \\
\mathbf{p}_{D}(n) & =\sum_{i=0}^{n} \lambda^{(n-i)} \mathbf{x}(i) d(i) .
\end{aligned}
$$

O fator de ponderação exponencial $(\lambda)$ é arbitrado tal que $0 \ll \lambda<1$.

\section{Procedimento EXPERIMENTAL}

\section{A. Conjunto de Dados}

O período do conjunto de previsão considerado é de 2008 a 2010, compreendendo intervalo de previsões entre 13 de Dezembro a 30 de Abril do ano seguinte. As séries utilizadas apresentam 139 dias de previsão e são denominadas de horizonte de previsão sazonal. As previsões Eta e reanálises NCEP apresentam resolução temporal de seis horas, portanto, por dia há quatro valores de previsão (às 00:00, 06:00, 12:00 e 18:00 UTC). Neste trabalho utilizamos o intervalo de 13 de Dezembro às 12 horas a 30 de Abril às 12 horas do ano seguinte, correspondendo a $P=553$ pontos em cada célula. Para todas as variáveis prognósticas foi adotado um filtro com quatro coeficientes (o que corresponde a um dia de previsão). Usamos os dados dos 2008 e 2009 para treinar filtros que são aplicados às variáveis prognósticas dos anos 2009 e 2010 , respectivamente.

As variáveis prognósticas utilizadas para a análise da proposta são as componentes zonal e meridional do vento (em $m / s$ ), altura geopotencial (em $m g p$ - metro geopotencial) e a umidade específica (em $\mathrm{kg}$ de massa de vapor d'água/ $\mathrm{kg}$ de massa de ar) [12]. A metodologia descrita na seção anterior é aplicada em dados correspondentes a vinte níveis de pressão atmosférica $(50 \mathrm{hPa}$ a $1000 \mathrm{hPa}$, com intervalo de $50 \mathrm{hPa}$, com exceção de $950 \mathrm{hPa}$ ). Porém, para a umidade específica não se aplica a proposta em níveis da alta troposfera (pressão $<250 \mathrm{hPa}$ ), onde a água é quase ausente e os valores dos coeficientes da DCT-2D são todos quase nulos.

\section{B. Configurações do RLS}

Em cada cenário (ano, variável prognóstica e nível de pressão), projetaram-se filtros adaptativos com diferentes valores de $\lambda$ para cada variável prognóstica. A Tabela I indica o valor de $\lambda$ que melhor auxiliou na obtenção de menores erros quadráticos após a filtragem adaptativa, para cada variável prognóstica, no restante deste trabalho. É importante frisar que os valores de $\lambda$ são para os anos de treinamento de 2008 e 2009 e os coeficientes resultantes dos filtros adaptativos são aplicados nos anos de ajuste de 2009 e 2010, respectivamente. 611 
TABELA I

FATORES DE PONDERAÇÃO EXPONENCIAL $(\lambda)$ UTILIZADOS PARA OS ANOS DE TREINAMENTO DE FILTROS ADAPTATIVOS.

\begin{tabular}{|c|c|c|c|}
\hline \multicolumn{2}{|c|}{ Variável prognóstica } & \multicolumn{2}{c|}{ Valores de $\lambda$} \\
\hline Nome Eta & Significado & $\mathbf{2 0 0 8}$ & $\mathbf{2 0 0 9}$ \\
\hline uvel & Comp. zonal do vento & 0.980 & 0.998 \\
\hline vvel & Comp. meridional do vento & 0.980 & 0.980 \\
\hline zgeo & Altura geopotencial & 0.990 & 0.990 \\
\hline umes & Umidade específica & 0.990 & 0.990 \\
\hline
\end{tabular}

\section{Critérios de Avaliação}

A partir do ajuste das séries temporais do modelo Eta pode ser definido o erro quadrático médio (MSE - Mean Square Error) entre as séries das reanálises do NCEP e as séries ajustadas do modelo Eta. Para cada nível de pressão atmosférica há um total de 38.763 séries temporais para o modelo Eta e também para o modelo NCEP, portanto, é possível calcular 38.763 valores de MSE em cada nível de pressão atmosférica.

Como exposto, lidamos com séries temporais correspondentes a valores climáticos em coordenadas geográficas específicas. Consequentemente, na região e na resolução do Eta analisado temos $I=38.763$. Utilizemos o subscrito $i$ para referenciar essas séries. Podemos definir o erro na $n$-ésima amostra da $i$-ésima série como

$$
e_{i}(n)=d_{i}(n)-y_{i}(n),
$$

na qual, $d_{i}(n)$ corresponde à série temporal do NCEP e $y_{i}(n)$ à do Eta. Assim, para cada célula $i$ podemos definir o erro quadrático médio (MSE)

$$
M S E_{i}=\overline{e_{i}^{2}}=\frac{1}{P} \sum_{n=1}^{P} e_{i}(n)^{2},
$$

onde a média é realizada no tempo e $P$ é o comprimento das previsões climáticas em amostras.

De forma a avaliar corretamente a proposta, faz-se necessário atentar para desvios extremos. Assim, avaliamos também o efeito da proposta sobre o erro máximo entre a previsão e o NCEP para as diferentes variáveis climáticas. Para esse fim, utilizamos o erro máximo absoluto

$$
e_{i, \text { máx }}=\max _{n}\left|e_{i}(n)\right| \text {. }
$$

A Figura 5 ilustra os comportamentos dos critérios acima. Nela vemos o MSE e o $e_{\text {máx }}$ da previsões Eta antes e após a aplicação de nossa proposta de redução de desvios, para a componente zonal do vento (uvel), ao nível de pressão atmosférica $300 \mathrm{hPa}$, considerando todo o ano de 2010. As regiões em tons mais escuros apresentam os menores valores

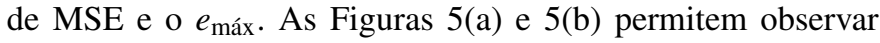
que há uma redução no MSE que é mais pronunciada em determinadas regiões, principalmente na América do Sul. Por sua vez, as Figuras 5(c) e 5(d) ilustram o comportamento do $e_{\text {máx }}$ para a mesma variável prognóstica, nível de pressão, região e período (ano de 2010). Neste caso, também observase uma redução do $e_{\text {máx }}$, isto é, dos erros extremos, em grande parte da região da América do Sul e as regiões adjacentes.

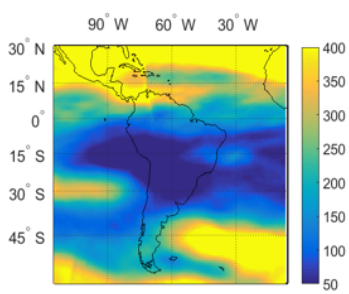

(a)

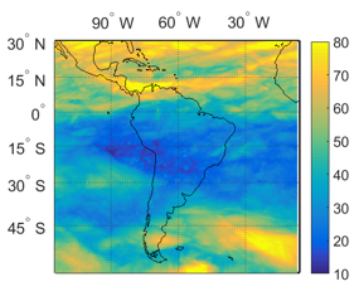

(c)

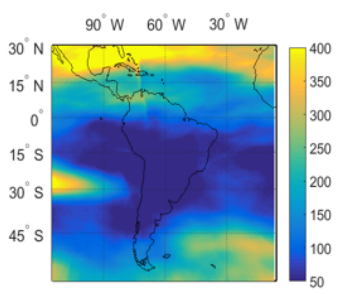

(b)

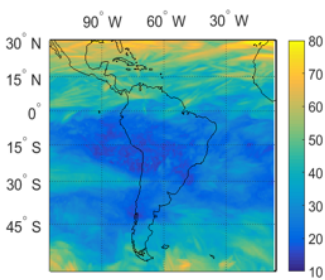

(d)
Fig. 5. Análise dos erros da previsão antes e após a utilização dos filtros propostos. Considera-se a componente zonal do vento $(\mathrm{em} \mathrm{m} / \mathrm{s})$ a $300 \mathrm{hPa}$ no ano de 2010: (a) MSE entre o Eta e o NCEP (em $\mathrm{m}^{2} / \mathrm{s}^{2}$ ), (b) MSE após o ajuste do Eta $\left(\mathrm{em} \mathrm{m}^{2} / \mathrm{s}^{2}\right)$; (c) $e_{\text {máx }}$ entre o Eta e o NCEP $(\mathrm{em} \mathrm{m} / \mathrm{s})$; e (d) $e_{\text {máx }}$ após o ajuste do Eta $(\mathrm{em} \mathrm{m} / \mathrm{s})$.

Dada a impossibilidade de apresentar resultados como os da Figura 5 para todas as variáveis prognósticas e níveis de pressão, esses dados são sintetizados em índices médios. Por exemplo, para um nível de pressão específico, a média do erro quadrático médio (MSEM) é

$$
M S E M=\overline{M S E_{i}}=\frac{1}{I} \sum_{i=1}^{I} \overline{e_{i}^{2}}=\frac{1}{I} \sum_{i=1}^{I} \frac{1}{P} \sum_{n=1}^{P} e_{i}(n)^{2},
$$

onde $M S E_{i}$ é o MSE da série temporal da variável climática na $i$-ésima coordenada geográfica.

A partir do MSEM pretendemos analisar se globalmente (em toda a região, $i=1, \ldots, I$ ) os filtros adaptativos para cada variável climática são ou não efetivos na redução do erro da previsão após suas aplicações. Isto é, se há redução dos valores de MSEM após o ajuste das séries temporais do modelo Eta. A redução percentual da média do erro quadrático médio $\left(M S E M_{p}\right)$ é expressa por

$$
\operatorname{MSEM}_{p}=\left(1-\frac{M S E M_{\mathrm{ap}}}{M S E M_{\mathrm{an}}}\right) \times 100,
$$

onde $M S E M_{\text {an }}$ e $M S E M_{\text {ap }}$ são, respectivamente, a média dos valores de MSE antes e após o ajuste das séries temporais do modelo Eta, em unidade de porcentagem.

Outra forma de avaliar o efeito geral da proposta é empregando uma "taxa de efetividade" $\left(T_{\mathrm{ef}}\right)$ : fração das séries temporais para as quais se consegue uma redução do erro de previsão numérica (avaliado, por exemplo, através do MSE ou do erro máximo) para os diferentes níveis de pressão atmosférica. Tal taxa seria definida por

$$
T_{\mathrm{ef}}=\left(\frac{N_{r}}{N_{s}}\right) \times 100,
$$

onde $N_{r}$ é o número de séries temporais nas quais se observou redução do índice avaliado (MSE ou erro máximo) e $N_{s}$ é o número total de séries em cada nível de pressão atmosférica. 


\section{RESUltados}

A Figura 6 apresenta os percentuais de redução no $\operatorname{MSEM}_{p}$. A metodologia proposta fornece resultados muito semelhantes para os dois anos estudados (2009 e 2010) para diferentes variáveis prognósticas (vvel, uvel, umes e zgeo) e níveis de pressão. As reduções observadas no $M S E M_{p}$ da altura geopotencial em 2009 e em 2010 são quase coincidentes, as outras, apesar de menos coincidentes, apresentam comportamentos bastante semelhantes nos dois anos. Com exceção de níveis de pressão específicos para os quais há maiores divergências, como por exemplo para a componente meridional do vento entre 100 e $250 \mathrm{hPa}$, vemos que o comportamento do $M S E M_{p}$ mostra-se correlacionado para uma mesma variável climática nos dois anos analisados.

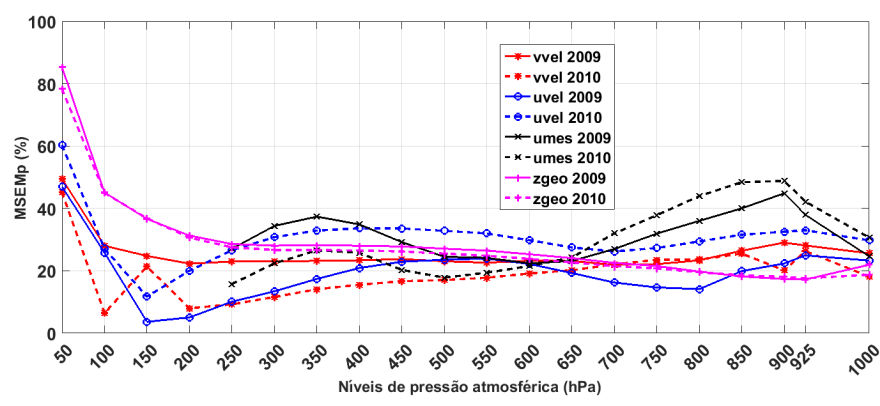

Fig. 6. Percentual de redução da média do erro quadrático médio $\left(M S E M_{p}\right)$ dos anos de ajuste de 2009 e 2010 , para a componente meridional do vento ( vvel), componente zonal do vento ( $u v e l$ ), altura geopotencial (zgeo) e umidade específica (umes).

A Figura 7 apresenta a taxa de efetividade na redução do MSE da previsão: a fração das séries temporais para as quais observa-se redução do MSE após o ajuste da previsão climática proposto. Através dessas curvas é calculada a taxa de efetividade média, que equivale a 76,77\%, indicando uma melhora geral do prognóstico climático. Com exceção de alguns percentuais de efetividade que apresentam uma queda abrupta, os comportamentos das taxas não sofrem oscilações acentuadas.

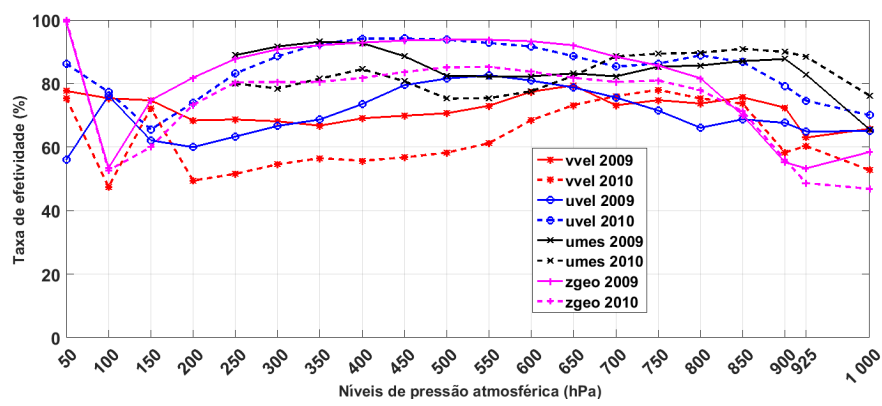

Fig. 7. Taxa de efetividade do MSE dos anos de ajuste de 2009 e 2010, para a componente meridional do vento (vvel), componente zonal do vento (uvel), altura geopotencial (zgeo) e umidade específica (umes).

A Figura 8 apresenta os percentuais de efetividade na redução do erro máximo da previsão correspondentes as variáveis prognósticas (uvel, vvel, umes e zgeo) nos anos de 2009 e 2010. Afora para a variável zgeo, a média dos percentuais de efetividade que reduzem o erro máximo das previsões climáticas é de $62,03 \%$, indicando um melhor comportamento do erro máximo para as previsões climáticas.

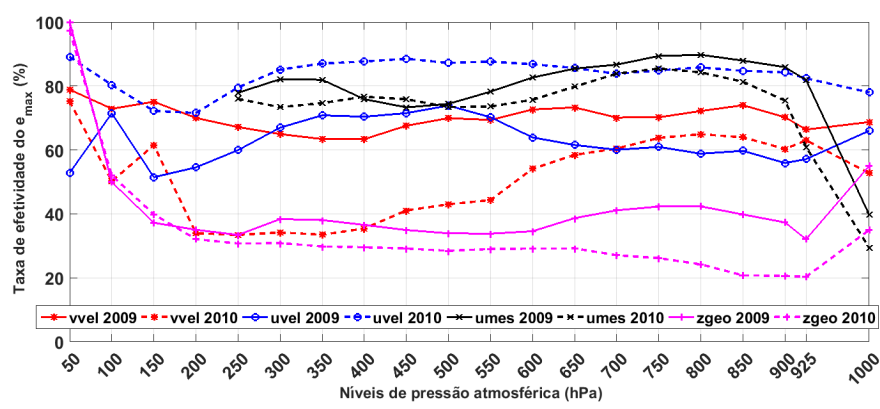

Fig. 8. Taxa de efetividade do erro máximo $\left(e_{\max }\right)$ dos anos de ajuste de 2009 e 2010, para a componente meridional do vento (vvel), componente zonal do vento (uvel), altura geopotencial (zgeo) e umidade específica (umes).

\section{CONCLUSÕES}

Neste trabalho propôs-se aplicar filtragem adaptativa aos coeficientes DCT-2D de previsão numérica climática de forma a reduzir os desvios da mesma. A filtragem dos componentes frequenciais objetivou capturar estruturas meteorológicas de diferentes períodos. Os filtros para correção da prognose são obtidos usando o algoritmo RLS. Definimos índices capazes de avaliar sinteticamente a qualidade da previsão em toda a região geográfica abordada pelo modelo climático. Com isso, observa-se que o uso de filtros adaptativos aplicados no domínio da frequência permite sistematicamente reduzir a energia do erro de previsão nos diferentes níveis de pressão atmosférica.

\section{REFERÊNCIAS}

[1] S. C. Chou, "Modelo regional eta, climanálise, edição comemorativa de 10 anos," INPE. São Jose dos Campos, SP, Brasil, 1996.

[2] A. R. Freitas, M. P. Tcheou, L. Lovisolo, and S. C. Chou, "Filtragem adaptativa para a redução de desvios em séries temporais de previsão numérica climática," Simpósio Brasileiro de Telecomunicações e Processamento de Sinais, 2015.

[3] F. Mesinger, K. Veljovic, S. C. Chou, J. Gomes, and A. Lyra, "The Eta model: Design, use, and added value," in Topics in Climate Modeling. InTech, 2016.

[4] S. C. Chou, J. F. Bustamante, and J. Gomes, "Evaluation of Eta model seasonal precipitation forecasts over South America," Nonlinear Processes in Geophysics, vol. 12, no. 4, pp. 537-555, 2005.

[5] NOAA/GFS, "Global forecast system," Disponível em: http://www.ncdc.noaa.gov/data-access/model-data. Acesso em: Março 2018.

[6] S. Saha, S. Moorthi, H.-L. Pan, X. Wu, J. Wang, S. Nadiga, P. Tripp, R. Kistler, J. Woollen, D. Behringer et al., "The ncep climate forecast system reanalysis," Bulletin of the American Meteorological Society, vol. 91, no. 8, pp. 1015-1058, 2010.

[7] P. S. R. Diniz, Adaptive filtering: Algorithms and Practical Implementation. Springer, 2013.

[8] S. S. Haykin, Adaptive filter theory. Pearson, 2008.

[9] P. S. R. Diniz, E. A. B. Da Silva, and S. L. Netto, Digital signal processing: system analysis and design. Cambridge University Press, 2010.

[10] G. D. Knott, Interpolating cubic splines. Springer Science \& Business Media, 2012.

[11] A. K. Jain, Fundamentals of digital image processing. Englewood Cliffs, NJ: Prentice Hall,, 1989.

[12] NOAA/NCEP, "Parameters and units," Disponível em: http://www.nco.ncep.noaa.gov/pmb/docs/on388/table2.html. Acesso em: Março 2018. 